\author{
Voix plurielles \\ Volume 4, Numéro 1 : mai 2007
}

Georges Farid

\title{
Accord ou invariabilité du participe passé : les règles problématiques
}

Citation MLA : Farid, Georges. «Accord ou invariabilité du participe passé : les règles problématiques.» Voix plurielles 4.1 (mai 2007).

(C) Voix plurielles, revue électronique de l'APFUCC 2007. 


\title{
Accord ou invariabilité du participe passé : les règles problématiques
}

\author{
Georges Farid \\ Université du Québec en Outaouais
}

Mai 2007

$\mathrm{L}$

'objectif de cette recherche descriptive est d'identifier, dans la multitude des règles du participe passé, celles qui présentent des cas problématiques. Par « problématique », il faut entendre les règles où existe une certaine flexibilité dans leur utilisation. Même si l'identification de ces règles est louable en soi, notre objectif ultime est non seulement de mettre en relief ces règles, mais aussi de cerner le pourquoi de cette flexibilité et ses conséquences dans la pratique évaluative, notamment dans le contexte scolaire.

Après l'analyse des règles du participe passé dans moult livres de grammaire, nous avons relevé neuf (9) règles d'accord avec l'auxiliaire " être », vingt-cinq (25) règles d'accord avec l'auxiliaire « avoir » et dix (10) règles d'accord avec les verbes pronominaux. Quant aux règles problématiques, nous avons repéré 17 cas qui, malgré les règles existantes, oscillent dans les fluctuations de la norme. Dans la section suivante, nous présentons chacun de ces cas avec les commentaires de différents grammairiens, commentaires que nous avons délibérément résumés, faute d'espace.

1- Le participe passé attribut du complément d'objet direct après les verbes dits d'opinion : considérer, croire, déclarer, élire, estimer, juger, nommer, préférer, proclamer, trouver, voir, etc.

- Grevisse (1980), bien qu'il note que la règle consiste à accorder le participe passé suivi d'un attribut d'objet direct si celui-ci précède le participe, souligne aussi qu'il n'est pas rare qu'on laisse ce participe invariable. Exemples : Une vie qu'on aurait VOULU belle (A. Maurois, Byron, XXVIII). L'armée qu'on avait CRU si forte (J. et J. Tharaud, Dingley, p. 190) (p. 919).

2- Le participe passé utilisé sans auxiliaire : cas de "admis, approuvé, attendu, autorisé, certifié, collationné, communiqué, compris, considéré, entendu, envoyé, eu égard à, examiné, excepté, expédié, fourni, lu, non compris, non compté, ôté, ouï, paraphé, passé, quitté, reçu, refait, signé, sous-entendu, soustrait, supposé, venu, vérifié, visé, vu, y compris »

- Grevisse (1980), malgré la règle qui veut que ces participes passés restent invariables lorsqu'ils sont placés avant le nom ou le pronom, note que « certains de ces participes [...], placés devant le nom, sont assez souvent considérés comme participes passifs, et s'accordent. Exemples : [...] PASSÉE la cinquantaine (Cl. Farrère, La Bataille, xII). [...] ÔTÉE la verve qui était exceptionnelle, il ne reste pas grand-chose (J. Green, Vers l'invisible, p. 130). » (p. 913).

- Hanse (1994), même s'il conseille l'invariabilité de « passé » avant le nom ou le pronom, 
note qu'on a le choix. Il cite, entre autres exemples, B. Clavel, qui tantôt accorde « passé » : PASSÉE la dernière maison (Le voyage du père, J'ai lu,

p. 219), tantôt le laisse invariable : PASSÉ les arbres (...) (L'Espagnol, J'ai lu,

p. 15, $311 »($ p. 638).

3-Le participe passé utilisé sans auxiliaire : cas de « ci-annexé, ci-annoncé, ci-épinglé, ci-inclus, ci-joint»

- Grevisse (1993), malgré la règle qui veut que ces participes passés s'accordent lorsqu'ils sont suivis, dans le corps de la phrase, d'un nom avec article ou avec déterminatif démonstratif, possessif, numéral ou indéfini, constate que « [...] l'usage n'est pas fixé, particulièrement quand, dans le corps de la phrase "ci-joint, ci-inclus, ci-annexé" précèdent un nom accompagné d'un déterminant :

Avec accord : [...] J'ai l'honneur de vous transmettre ci-JOINTES la réclamation de M. le Capitaine, ma lettre à M. le Délégué et sa réponse (Stendhal, Corresp. T. viII, p. 324-325)

Sans accord : [...] J'ai l'honneur de vous envoyer ci-INCLUs la déclaration que vous me demandez (Hugo, Corresp. Cit. Trésor, [...]).» (p. 1332).

4- Le participe passé utilisé sans auxiliaire : cas de "étant donné, mis à part, fini »

- Grevisse (1980) mentionne que « l'expression ÉTANT DONNÉ peut aujourd'hui être assimilée aux participes "attendu, compris, etc. " et rester invariable quand elle précède le nom : ainsi traitée, elle joue le rôle d'une préposition; mais l'accord de DONNÉ avec le nom ou les noms qui suivent reste très correct : [...] ÉTANT DONNÉ les mœurs françaises du temps (La varende, Les Belles Esclaves, p. 102) [...] ÉTANT DONNÉES les circonstances présentes (Saint-Éxupéry, Pilote de guerre, p. 15). » (p. 914).

- Grevisse (1993) cite aussi : «MISES à part les professionnelles et quelques folles (Yourcenar, Souvenirs pieux, p. 268). [...] MIS à part une soixantaine de personnes (Le Roy Ladurie, Carnaval de romans, p. 28-29)» (p. 361).

- Hanse (1994) souligne que « fini » s'accorde généralement mais peut rester invariable. Exemples : FINIES les vacances (= les vacances sont finies). FINI les vacances (= C'est fini les vacances) (p. 638).

5- Le participe passé avec « avoir» dans les locutions verbales « l'échapper belle, la manquer belle, la manquer bonne, la bailler belle, la bailler bonne »

- $\quad$ Grevisse (1996) indique que le participe passé reste invariable dans ces expressions. Cependant, il note que « ces mêmes expressions, selon G. et R. Le Bidois, il paraît licite, et même recommandable de les écrire au féminin, c'est-à-dire, “ ÉCHAPPÉE, BAILLÉE, MANQUÉE” (p. 21).

6- Le participe passé avec « avoir 》 et antécédents joints par «ou, ni »

- Grevisse (1980) cite Girault-Duvivier (t. I, p. 580) pour qui le dernier antécédent est le seul qui commande l'accord parce qu'il n'y a pas addition, mais disjonction. Exemple : Est-ce une poire ou deux poires qu'il a MANGÉES (p. 924).

- $\quad$ Grevisse (1980) note que l'usage pratique laisse instinctivement le participe invariable. Exemple : Quel poison ou quelle drogue m'a-t-on INJECTÉ, qui m'incendie? (J. Roy, Le Maître de la Mitidja, p.253) (p. 924).

7-Le participe passé avec « avoir » et antécédent adverbe de quantité ou locution de quantité : 
assez de, autant de, beaucoup de, bien de, combien de, le peu de, moins de, nombre de, pas mal de, peu de, plupart de, plus de, quantité de, que de, tant de, tellement de, trop de...

- Grevisse 1980 note que « lorsqu'un participe passé à accorder est en rapport avec un collectif (ou un nom de fraction) suivi de son complément, l'accord est commandé par le collectif (ou par le nom de fraction) ou par le complément suivant que l'esprit, d'après le sens de la phrase ou l'intention, est plus particulièrement frappé par l'un ou par l'autre :

[...] La moitié des côtes FRACTURÉES (L. Bloy, Le Désespéré ， p. 433). - Plus de la moitié du travail était TERMINÉE (H. Troyat, Dostoïevsky, p. 277). » (p. 921).

- Grevisse (1996), bien qu'il souligne que le participe passé en rapport avec un adverbe de quantité accompagné de son complément s'accorde avec ce complément, note aussi qu'il arrive que l'adverbe de quantité domine dans la pensée et commande l'accord. Donc, on pourra accepter aussi bien « Trop de haine lui fut TÉMOIGNÉE » (accord du participe passé avec le complément de l'adverbe au féminin singulier) que « Trop de haine lui fut TÉMOIGNÉ » (le participe passé reste invariable parce que l'accord avec l'adverbe de quantité « trop » reste au masculin singulier). Il en est ainsi avec les autres adverbes de quantité « le peu de, trop de, tant de » (p. 25).

8- Le participe passé avec " avoir 》 et " dormir, durer, marcher, régner 》

Bien que la règle veuille que les participes passés de ces verbes soient invariables, Grevisse (1993) note que « le participe passé DORMI est parfois employé au passif : Trois nuits mal DORMIES (Musset, Prem. Poés. Marrons du feu, IV). - Las d'une nuit mal DORMIE (Vian, Arrache-cœur, IX). [...] Dès lors, il ne serait pas impossible d'écrire : Les trois nuits que j'ai mal DORMIES. Mais cela reste de la pure théorie » (p. 1337).

9- Le participe passé avec " avoir » et « courir, coûter, dépenser, distribuer, donner, gagner, mesurer, parier, perdre, peser, prendre, rapporter, recevoir, recueillir, récolter, régner, travailler, valoir, vivre... "

Selon la règle, le participe passé des verbes « courir, coûter, mesurer, peser, valoir,

vivre » conjugués avec « avoir » reste invariable parce que, pris au sens propre, son complément, même s'il précède le verbe, est un complément circonstanciel et non pas un complément d'objet direct.

- Grevisse (1980) note que, pour Littré, « coûté » doit toujours rester invariable, que le sens soit propre ou figuré. Exemples : La somme que cette maison m'a COÛTÉ. Les pleurs que la mort de cet enfant a coûté à sa mère (p. 916).

- Grevisse (1993), avec le verbe « vivre », donne des exemples où l'accord ou le non-accord sont possibles dans une perspective particulière : «VIVRE est considéré par beaucoup d'auteurs comme un verbe transitif signifiant "passer, mener" quand on envisage, non pas la durée en ellemême, mais la nature, la qualité de ce qui a été expérimenté au cours de cette période (qualité souvent exprimée par une épithète, un complément déterminatif, etc.). [...] : Les heures qu'il avait VÉCUES loin de Dieu (France, Thaïs, p. 11) [...] Ce ne sont pas des heures frivoles que j'aurais VÉCUES (Mauriac, Journal, t. III, p. 111).

Même dans ce cas, certains auteurs répugnent à considérer qu'il s'agit d'un véritable objet direct : Quelles étranges minutes elle avait VÉCU! (Green, Léviathan, I, 9.) [...] Pour que les temps qui viennent nous soient moins amers [...] que ceux que nous avons VÉCU en 1940 (Valéry, Variété, P1., p. 883). » (p. 1337). 


\section{0- Le participe passé avec « avoir » et suivi de l'infinitif}

Malgré la règle qui veut que le participe passé suivi de l'infinitif, d'une part, s'accorde si le complément d'objet direct qui précède se rapporte à ce participe et fait l'action et, d'autre part, ne s'accorde pas si le complément d'objet direct qui précède ne se rapporte pas au participe (mais à l'infinitif) et ne fait pas l'action, Bescherelle (1998) souligne que l'Arrêté Haby de 1976 tolère les deux possibilités dans tous les cas (paragraphe 139). Il est à noter que cela ne s'applique pas au participe passé de « faire » suivi d'un infinitif, auquel cas « fait» reste invariable.

11- Le participe passé avec " avoir » et « donné, eu, laissé suivis de "à » et de l'infinitif

- Grevisse (1996) - malgré la règle reliée à cette notion et le sens qui imposerait, soit l'invariabilité, soit l'accord du participe passé - note que « on a le choix entre l'accord et l'invariabilité du participe. Exemples : Les ennemis que j'ai EUS à combattre. Les ennemis que j'ai EU à combattre (p. 32).

- Grevisse (1993) ajoute, dans la même perspective, plusieurs exemples dont : La leçon que je lui ai DONNÉE à étudier (Ac.). [...] Je fus tellement pris par ces vers que l'on m'avait DONNÉ à apprendre que [...] (Jammes, De l'âge divin à l'âge ingrat,

p. 117). La seule turpitude que les doctrinaires et républicains lui eussent LAISSÉ à désirer (Bloy, Désespéré, p. 341). Les problèmes qu'il nous a LAISSÉS à résoudre (Salacrou, Archipel Lenoir, II). » (p. 1342).

- $\quad$ Boudou (1995) note : «Le plus souvent il y a ambiguïté, si bien que le double usage est admis. Exemple : Les leçons que j'ai Eu(ES) à apprendre étaient difficiles. » (p. 43).

- Hanse (1994) constate également le double usage mais conseille de laisser le participe passé invariable quand le sens est « devoir ». Exemple : Les livres qu'on nous a DONNÉ(S) à lire (p.646).

12- Le participe passé avec « avoir » et «fait, laissé » suivis de l'infinitif

- $\quad$ Boudou (1995), malgré la règle qui veut que le participe passé « laissé » s'accorde avec le complément d'objet direct qui le précède si celui-ci fait l'action, se réfère

à l'article « Les rectifications de l'orthographe » publié le 6 décembre 1990 dans le Journal officiel par le Conseil supérieur de la langue française, qui conseille

« de garder le participe passé "laissé" suivi d'un infinitif toujours invariable ».

- Girodet (1988) note : « Dans l'usage moderne, on rencontre souvent "laisser" invariable dans des cas où la règle exigerait l'accord. » (p. 187).

- Christensen et al. (1995) notent que « selon la nouvelle réforme de l'orthographe, le participe passé de "laisser" suivi d'un infinitif est rendu invariable dans tous les cas. Exemples : Elle s'est LAISSÉ aller. Je les ai LAISSÉ partir. » (p. 14).

- Goosse (1991) statue sur ce litige : «Le participe passé "laissé” suivi d'un infinitif reste invariable. Exemples : Tes poissons rouges, tu les as LAISSÉ mourir de faim. » Goosse souligne que « on observe un double usage, et, pour éviter le désordre que craignent aussi les correcteurs, le Conseil a transformé la latitude en règle » (p. 70).

13- Le participe passé avec " avoir » et précédé du pronom " en »

- $\quad$ Christensen et al. (1995) notent que, à part la règle évidente qui veut que le participe passé précédé de « en » s'accorde lorsqu'il ne se rapporte pas à ce pronom neutre complément d'objet indirect mais bien au complément d'objet direct qui précède, « selon la plupart des grammairiens, 
le participe passé précédé de « en » reste invariable. Néanmoins, dans l'usage, l'accord a souvent lieu. Exemple : J'ai ramassé des crevettes. J'en ai RAMASSÉ(ES). » (p. 15).

- Grevisse (1996) note qu'on a le choix entre l'invariabilité du participe passé comme dans «Voyez ces fleurs, en avez-vous CUEILLI? (Littré) » et l'accord du participe passé comme dans «Les fleurs, il n'en avait jamais VUES (M. Proust). » (p. 26).

14- Le participe passé des verbes pronominaux réfléchis avec un complément d'objet direct invisible

- Grevisse (1996), malgré la règle qui permet l'accord du participe passé avec le complément d'objet direct (invisible) si celui-ci est placé avant le participe passé, mentionne que « avec "se persuader que, s'assurer que" (= être certain), l'accord du participe passé est facultatif. Exemple : Elles se sont PERSUADÉ (ES), elles se sont ASSURÉ(ES) que l'occasion était bonne » (p. 34, $\mathrm{n}^{0}$ 3).

- Hanse (1994), malgré la règle qui exige l'invariabilité de la locution pronominale « se faire fort de » dans le sens de « se déclarer capable de, s'engager à, se

vanter », confirme que "l'usage s'est établi de laisser "fort" invariable [...] et même d'y laisser invariable le participe "fait" : Elle s'est FAIT FORT de la convaincre (Ac.) ». Cependant, Hanse note que des écrivains font quand même l'accord (p. 411).

15- Le participe passé des verbes pronominaux sans complément d'objet direct

- Grevisse (1993), malgré l'invariabilité du participe passé des quatre verbes « se complaire, se déplaire, se plaire, se rire », note : " Il y a dans l'usage une tendance à accorder " plu, complu, déplu " comme le participe des autres pronominaux subjectifs : Chez tous elle s'était PLUE à éveiller l'amour (Maurois, Chateaubriand, p. 294) [...] Elle venait de 1'“Aigue " où elle s'est DÉPLUE (Veuillot, Corresp. t.II, p. 462 [...] Vous êtes-vous compLue à ces pensées ? (Mistler, Route des étangs, p. 83). » (p. 1344-1345).

16- Le participe passé des verbes pronominaux suivis de l'infinitif

- Malgré la règle qui exige que le participe passé reste invariable si le sujet n'accomplit pas l'action exprimée par l'infinitif, Girodet (1988) note que « cette règle n'est pas toujours appliquée, même par les bons écrivains modernes. »

(p. 204).

- Hanse (1994) cite, dans le sens de la remarque précédente de Girodet, plusieurs exemples dont : « Nous nous sommes LAISSÉS entraîner de nouveau (...). Nous nous sommes LAISSÉS séduire (A. Gide, Journal, 20 février 1928, 23 avril 1932). Elle s'était LAISSÉE marier docilement à un vieillard (Fr. Mauriac, Le nœud de vipères, $1^{\text {re }}$ partie, ch. vIII). » (p. 652).

- Goosse (1991) statue sur ce litige : « Le participe passé "laissé” suivi d'un infinitif reste toujours invariable. Exemple : Ils se sont LAISSÉ mourir de faim.» (p.70).

17- Le participe passé des verbes pronominaux suivis d'un attribut du pronom réfléchi complément d'objet direct

- Grevisse (1980), malgré la règle qui permet l'accord du participe passé, note que « puisqu'il n'est pas rare qu'on laisse le participe invariable dans des phrases du type "Ces personnes, je les ai CRU mortes" [...], on pourrait écrire aussi "Elles se sont CRU belles " » (p. 936).

- Grevisse (1982), malgré la règle qui permet l'accord du participe passé et avec laquelle il est en accord, note que « Hanse, Thomas, Robert, Le Grand Larousse de la langue française 
tiennent que, pour "se faire l'écho de", le participe "fait" est invariable. Mais on ne voit pas pourquoi il ne pourrait pas être variable $[\ldots]$ : Les Goncourt se sont FAITS l'écho de certaines de ses confidences (A. Billy)»

(p. 290).

- Hanse (1994) partage plus tard la même opinion que Grevisse : "“Se faire l'écho de" est considéré par certains dictionnaires comme une expression plus ou moins figée où le participe est invariable : Ils se sont FAIT l'écho de la nouvelle (Le Grand Robert). Elle s'en est FAIT l'écho (Le Grand Larousse de la langue française). Mais la logique et l'usage autorisent - et même recommandent - l'accord, comme on le ferait dans "Elle s'est FAITE la protectrice des réfugiés [...]" » (p. 326). Hanse (2000), dans la quatrième édition, maintient la même opinion (p. 217).

\section{Essai explicatif de l'accord ou de l'invariabilité du participe passé}

Voilà 17 cas où le participe passé oscille dans les fluctuations de la norme. Comment expliquer ce que certains caractérisent comme « caméléon grammatical » et d'autres comme « flou grammatical », quelles que soient les conséquences pour les scripteurs.

L'analyse des cas précédents nous permet d'observer un leitmotiv sémantique dont la forme structurale varie : «Bien que... il n'est pas rare que, malgré que...on a le choix, l'usage n'est pas fixé, le double usage est permis, l'accord est facultatif, malgré... il y a dans l'usage une tendance à... ».

Dans la plupart de ces cas, la justification à la fois de l'accord et de l'invariabilité est fondée sur des illustrations de la littérature ou d'écrivains notoires. Un seul cas (cas $\mathrm{n}^{0} 12$ Le participe passé avec " avoir » et "fait, laissé » suivis de l'infinitif) semble s'affirmer, et ce, en référence à l'article "Les rectifications de l'orthographe » publié le 6 décembre 1990 dans le Journal officiel par le Conseil supérieur de la langue française, qui conseille « de garder le participe passé "laissé" suivi d'un infinitif toujours invariable », qu'il s'agisse d'un emploi pronominal ou non pronominal.

Il est intéressant de noter, en plus des références aux écrivains, le cas $\mathrm{n}^{0} 7$ (Le participe passé avec " avoir » et antécédent adverbe de quantité ou locution de quantité) où Grevisse donne un commentaire relié à l'intellect : « l'accord est commandé par le collectif (ou par le nom de fraction) ou par le complément suivant que l'esprit, d'après le sens de la phrase ou l'intention, est plus particulièrement frappé par l'un ou par l'autre. »

Un dernier cas, cas $\mathrm{n}^{0} 10$ (Le participe passé avec " avoir » et suivi de l'infinitif) mérite une remarque particulière. En effet, malgré la règle bien précise et à l'exception de « faire » suivi d'un infinitif dont le participe passé doit rester invariable, Bescherelle (1998) a recours à l'Arrêté Haby de 1976 pour souligner les deux possibilités de l'accord et de l'invariabilité dans tous les cas (paragraphe 139). Toutefois, nous estimons ce genre de référence discutable, voir inapproprié pour les raisons que nous présenterons ultérieurement dans le contexte scolaire.

La réponse au pourquoi de cette flexibilité des 17 cas précédents se résume tantôt à une explication d'ordre intellectuel (cas $\mathrm{n}^{0} 7$ ), tantôt en référence à l'usage, notamment celle d'illustres écrivains incontestables. Cette maigre constatation nous a amené à remonter à l'histoire de la langue française dans l'espoir de trouver une explication. Le résultat est surtout relié à des aspects, encore une fois, d'usage et, à un moindre degré,

linguistiques: 
- Nathalie Fournier (1999) note que « en ancien et en moyen français le participe s'accorde avec le complément direct, quelles que soient leurs positions respectives; cependant se dégage déjà une très forte tendance à l'invariabilité du participe dans les séquences avoir + ppé + GN [...]. Cette tendance [...] aboutit à la règle, au XVIe siècle, de Marot, qui fonde notre norme écrite, selon laquelle le participe passé s'accorde avec le complément direct s'il est antéposé; cette règle fut minutieusement examinée au XVII siècle et complétée par des consignes, dont la complexité et les contradictions tiennent à ce qu'entrent en jeu plusieurs facteurs :

a) un strict facteur de position, qui fonde la norme graphique : le participe s'accorde avec l'objet antéposé (c'est la règle de Marot);

b) un facteur phonétique : le participe s'accorde quand il est en position finale dans l'énoncé et ne s'accorde pas s'il est suivi de constituants (sujet, attribut, groupe prépositionnel) : la peine qu'il m'a donnée /vs/ la peine que m'a donné cette affaire. [...] l'unanimité ne se fait d'ailleurs pas parmi les classiques sur la validité de ce critère, pris en compte par Vaugelas et Bouhours, mais contesté par Thomas Corneille;

c) un facteur syntaxique : le participe tend à l'invariabilité dans [...] les constructions dans lesquelles le GN est complément de l'infinitif régime et non du verbe auxilié : cette femme je l'ai fait peindre, c'est une fortification que j'ai appris à faire. »

Par rapport à nos cas problématiques énumérés précédemment, nous remarquons que dans le cas $\mathrm{n}^{0} 12$ (Le participe passé avec " avoir » et «fait, laissé » suivis de l'infinitif), il semble y avoir unanimité, depuis le XVII siècle, à laisser le participe passé invariable s'il est suivi de l'infinitif « faire », alors que les opinions sont partagées dans les autres cas : « [...] l'invariabilité du participe, demandée par Vaugelas et Bouhours, est refusée par Th. Corneille. Dans l'usage, non-accord et accord coexistent : [...] Tantôt à son aspect je I'[= Athalie] ai vu s'émouvoir (Racine, Ath., 1618) $-1691 ;[. .$.$] Il l'a laissée trop vivre après la mort de l'empereur Maurice son mari (Corneille, Hébr.,$ Examen, II, 359)- 1660. »

- Vaugelas (1647) donne ce qui sera, en grande partie, la base de nos règles participiales d'aujourd'hui, lesquelles nous présentons en résumé pour exposer le raisonnement sous-jacent (en gardant l'orthographe de l'époque) :

«[...] Notez que participes \& preterits ne sont qu'une mesme chose.

Premierement, le preterit va devant le nom qu'il regit, comme quand je dis, j'ay receu vos lettres. Alors receu, qui est le participe, est indeclinable.

Son second usage est, quand le nom va devant le preterit, comme quand je dis, les lettres que j'ay receües; car alors il faut dire, que j'ay receües; \& non pas que j'ay receu, à peine de faire un solecisme. Cela est passé en reigle de Grammaire, non seulement aujourd'huy, mais du temps mesme d'Amyot, qui l'observe inviolablement; comme on faisoit desja du temps, \& avant le temps de Marot, qui en a fait cette Epigramme à ses disciples : [...] Nostre langue a cette façon que le terme qui va devant, volontiers regit le suivant. [...]

Vaugelas ajoute une remarque qui ne devrait pas nous surprendre : « Neanmoins je m'estonne de plusieurs autheurs moderne, qui faisant profession de bien escrire, ne laissent pas de commettre cette faute.

En troisiesme lieu, le preterit peut estre placé entre deux noms, comme les habitans nous ont rendu maistres de la ville; car ont rendu est un preterit situé entre deux noms, à sçavoir nous, (que j'appelle 
nom, quoy qu'il soit pronom, parce que cela n'importe) \& maistres, qu'il regit tous deux à l'accusatif. Alors le participe est indeclinable [...] Mais il faut prendre garde [...] le preterit ont rendu ne finit pas la periode, ny le sens, car il y a encore aprés maistres de la ville. C'est pourquoy l'usage du preterit estant different, il se gouverne d'une autre façon, \& maistres qui le suit, marque assez le pluriel, sans qu'il soit besoin que le participe le marque encore.

En quatriesme lieu, le preterit estant placé entre deux noms, le dernier est, ou substantif comme maistres, [...], ou adjectif, qui fait que le quatriesme usage, par exemple, le commerce nous a rendu puissants, \& si nous parlons d'une ville, le commerce l'a rendu puissante; car en ces exemples il est indeclinable, \& ne suit ny le nommmbre ny le genre des noms.

Son cinquiesme usage, est quand le preterit est passif; [...] par exemple, nous nous sommes rendus maistres, ou rendus puissans. Alors il faut dire rendus, \& non pas rendu, ce participe dans le preterit passif n' estant plus indeclinable, mais prenant le nombre \& le genre des noms qui le precedent \& le suivent. [...] Il y a une exception, quand après le preterit passif il y a un participe passif, comme en cét exemple de M. de Malherbe, la desobeissance s'est trouvée montée au plus haut point de l'insolence, car il faut dire, s'est trouvé montée, \& non pas s'est trouvée montée. [...]

Son sixiesme usage est, quand les preterits actifs, ou passifs, au lieu d'un nom, ont un verbe en suite; car alors ils sont tousjours indeclinables sans exception, comme si je parle d'une fille je diray, je l'ay fait peindre, \& non pas, je l'ay faite peindre [...].

Ce mesme usage s'estend encore aux phrases, où entre le preterit \& le verbe infinitif qui suit, il y a quelque mot, comme, c'est une espece de fortification que j'ay appris à faire en toutes sortes de places, \& non pas que j'ay apprise à faire. La raison de cela [...] est qu'il faut aller en ces sortes de phrases jusqu'au dernier mot qui termine le sens, \& que par consequent c'est tousjours le dernier mot des phrases entiesres, qui a rapport au substantif precedent, $\&$ non pas le participe, qui est entre deux [...]. »

- Vaugelas donne ensuite dix exemples pour illustrer les règles ou les divers usages qu'il commente :

I. J'ay receu vos lettres. II. Les lettres que j'ay receües. III. Les habitans nous ont rendu maistres de la ville. IV. Le commerce, parlant d'une ville, l'a rendu puissante. V. Nous nous sommes rendus maistres. VI. Nous nous sommes rendus puissans. VII. La desobeissance s'est trouvé montée au plus haut point.VIII. Je l'ay fait peindre, je les ay fait peindre. IX. Elle s'est fait peindre, ils se sont fait peindre. X. C'est une fortification que j'ay appris à faire.

Le premier \& le second exemple sont sans contredit. Le troisiesme, quatriesme, cinquiesme, sixiesme, \& septiesme, sont contestez, mais la plus commune \& la plus saine opinion est pour eux. Le huitiesme, neuviesme, $\&$ dixiesme, ne reçoivent point de difficultlé, toute la Cour $\&$ tous nos bons Autheurs en usent ainsi. (pp. 175-181).

- Bouhours (1675), 28 ans après Vaugelas, se situe dans la même perspective que ce dernier en justifiant quelques règles en référence au latin et à la phonétique (nous les résumons, avec l'orthographe de l'époque) :

[...] Avec le verbe avoir, il (le participe passé) est naturellement indeclinable, n'ayant ni genre, ni nombre. J'ay receû vos Lettres; j'ay receû vos livres, parce que c'est plûtôt le supin des Latins, que le participe; \& que c'est comme si on disoit, habeo acceptum litteras, habeo acceptum libros.

[...] Mais il y a une autre raison qui oblige de parler d'une autre manière; \& c'est lors que la 
prononciation ne seroit pas assez soutenë. Car en ces rencontres, on donne des nombres $\&$ des genres aux participes, afin de soûtenir le discours. On dit pour cela, la lettre que j'ay receûe, la liberté que j'ay prise [...] Cela est si vray, que lors qu'on ajoûte quelque chose après, le participe redevient indéclinable, estant suffisamment soûtenu par ce qui suit, comme il paroist dans les exemples de M. de Vaugelas [...] (pp. 468-471).

- Corneille (1687), quant à lui, s'oppose à certaines règles de Vaugelas :

[...] je suis du nombre de ceux qui contestent quelques exemples de ceux qui sont rapportez [...] \& que je ne le fais qu'en suivant les sentiments de plusieurs personnes qui savent tres-bien écrire. Dans ceux-cy, les habitans nous ont rendu maistres de la ville; le commerce (parlant d'une ville) l'a rendu puissante. M. de Vaugelas dit que le participe est indéclinable. [...] il ne me paroist aucune raison qui me fasse croire qu'il faille dire, nous ont rendu maistres de la ville, \& non pas rendus, parce que le preterit ont rendu, ne finit pas la periode ny le sens, \& qu'on trouve encore après maistres de la ville. [...] (in Commentaires Vaugelas, t. I, pp. 359-361).

- Bescherelle frères et Litais de Gaux (1836) pour trancher certains cas litigeux ne se gênent pas d'avoir recours à des arguments comme : « Toutes les fois que les nuances deviennent trop délicates, l'analogie seule peut instruire, et l'instinct dirige mieux que le raisonnement » (p. 679). Cette citation, bien qu'elle soit issue de l'interprétation de l'opposition des formes du gérondif et du participe de l'époque, nous la ramenons dans le contexte du participe passé pour montrer que lorsque les nuances deviennent trop délicates, Grevisse (1996) évoque un argument similaire comme dans notre cas $\mathrm{n}^{0} 7$ précité : « ... il arrive que l'adverbe de quantité domine dans la pensée et commande l'accord » ou «... l'accord est commandé par le collectif (ou par le nom de fraction) ou par le complément suivant que l'esprit, d'après le sens de la phrase ou l'intention, est plus particulièrement frappé par l'un ou par l'autre ».

- Grevisse (1980), par rapport au cas $\mathrm{n}^{0} 2$ Le participe passé utilisé sans auxiliaire : cas de " admis, approuvé, attendu, autorisé, certifié, collationné, communiqué, compris, considéré, entendu, envoyé, eu égard à, examiné, excepté, expédié, fourni, lu, non compris, non compté, ôté, ouï, paraphé, passé, quitté, reçu, refait, signé, sous-entendu, soustrait, supposé, venu, vérifié, visé, $v u, y$ compris », signale que «dans l'ancienne langue, attendu, excepté, vu, etc. placés devant le nom, étaient tantôt laissés invariables, tantôt accordés; c'est à partir de la fin du moyen âge que l'usage s'est établi de les laisser invariables. Il subsistait cependant une tendance à les accorder : Il n'est pas apparent que il se fust mis en peril pour si peu de chose, VEÜES les offres qui luy avoyent esté faictes (Commynes, t. II, p. 105). PASSÉE la mer Picrocholine (Rabel., Garg., 33). EXCEPTÉE la nacelle où estoient ces deux petits enfans (Amyot, Romul., I). De nos jours encore certains de ces participes (notamment PASSÉ), placés devant le nom, sont assez souvent considérés comme participes passifs, et s'accordent : Mais, PASSÉES les épreuves des débuts, la troupe prend figure d'entreprise qui prospère (R. Doumic, Le Misanthrope de Molière, p. 28) [...]. »

- Grevisse (1980), par rapport au cas $\mathrm{n}^{0} 13$ Le participe passé avec " avoir » et précédé du pronom « en » note que « que ce pronom fût ou non complément d'un adverbe de quantité, l'accord était autrefois facultatif : [...] Combien en as-tu VU, je dis des plus huppés (Id., Plaid., I, 4) [...] Je ne veux pas vous faire pitié, puisque vous n'en avez pas déjà EUE pour moi (Rac., t. vI, p. 385, note 3) (p. 934).

Nous avons tenu à exposer, bien qu'en résumant beaucoup, ce que les grammairiens de 
l'époque notaient afin de déceler le raisonnement susceptible d'expliquer les règles du participe passé d'aujourd'hui, celles considérées bien établies, et celles que nous avons appelées « cas problématiques » ou cas litigieux. Nous constatons que reviennent quelques justifications fondées sur une analyse grammaticale qui varie selon la perception de tel ou tel grammairien, et ce, avec recours à l'usage d'écrivains renommés, sinon à l'impressionnisme grammatical comme dans le cas $\mathrm{n}^{0} 7$.

Il n'est pas étonnant que nous soyons aujourd'hui les héritiers des enseignements des grammairiens de l'époque en ce qui concerne l'analyse grammaticale, même si ceux-ci ne s'entendaient pas sur toutes les notions en question. À titre d'exemple pour illustrer ce propos, citons Chaurand (1999) qui fait référence au plan de direction d'études du collège de la ville d'Orléans, comme celui de la ville de Lyon et de bien d'autres villes, qui stipule en 1804 : « [...] Les ouvrages les plus propres pour apprendre les éléments de la langue françoise sont la Grammaire de Restaut, les Observations de Vaugelas, avec les notes de Th. Corneille, celles de l'Académie françoise, celles du P. Bouhours [...]» (p. 464).

Quant à l'impressionnisme grammatical, expression que nous empruntons à chaurand, celle-ci s'explique chaque fois que des grammairiens comme Bescherelle frères et Litais de Gaux (1836) se justifient comme suit : «Toutes les fois que les nuances deviennent trop délicates, l'analogie seule peut instruire, et l'instinct dirige mieux que le raisonnement. [...] D'ailleurs, en ceci comme en toute autre chose, l'oreille, le goût, l'harmonie, et quelquefois l'énergie peuvent seuls déterminer la place que doit, en certaines circonstances, occuper le pronom [...]» (p.465).

Dans le même ordre d'idées, Dauzat (1967) nous apprend que « les études de grammaire datent, en France, du XVIe siècle [...] la première grammaire rédigée par un Français fut écrite en latin [...] Le XVII siècle a fourni un gros effort : dans les salons et les cercles littéraires, à l'Académie dès sa fondation, les discussions grammaticales tiennent une place importante. Deux noms émergent : ceux de Vaugelas (Remarques sur la langue françoise, 1647) et de Ménage (Observations sur la langue françoise, 1672), qui, l'un et l'autre, cherchent à fonder les règles à la fois sur le "bel usage" et sur la raison $\gg($ p.141).

En ce qui concerne le cas $\mathrm{n}^{0} 2$ Le participe passé utilisé sans auxiliaire : cas de " admis, approuvé, attendu, autorisé, certifié, collationné, communiqué, compris, considéré, entendu, envoyé, eu égard à, examiné, excepté, expédié, fourni, lu, non compris, non compté, ôté, ouï, paraphé, passé, quitté, reçu, refait, signé, sous-entendu, soustrait, supposé, venu, vérifié, visé, vu, y compris », Dauzat souligne que « l'emploi des participes passé (attendu, excepté, vu...) est dû à la langue du droit et des chancelleries. Le français s'est ainsi créé, pour préciser les divers rapports [...] au XVII siècle » (p.185).

Aucune explication n'est donnée sur le pourquoi de la flexibilité de ce cas aujourd'hui.

Par rapport au cas $\mathrm{n}^{0} 10$ Le participe passé avec " avoir » et suivi de l'infinitif, Dauzat note que «Les grammairiens ont accepté l'invariabilité du participe dans le groupe fait faire, où le premier terme est un auxiliaire [...] » (p. 210). Sur ce point, il semblerait que les grammairiens se soient accordés, depuis très longtemps, à ne pas accorder le participe passé en question puisqu'ils le considèrent comme un auxiliaire.

Quant au cas $\mathrm{n}^{0} 14$ Le participe passé des verbes pronominaux réfléchis avec un complément d'objet direct invisible, Dauzat souligne que « le participe passé des verbes réfléchis, en ancien 
français, s'accordait généralement avec le sujet, accord conservé en principe (elle s'est piquée), sauf lorsque le verbe a un régime direct (elle s'est piqué le doigt), auquel cas les grammairiens, pour des raisons d'ordre logique, ont assimilé ces verbes aux verbes transitifs conjugués avec "avoir" (la maison qu'il s'est construite) [...]» (p. 210). Cependant, rien ne justifie le double emploi des pronominaux « s'assurer que, se persuader que » à moins que certains auteurs, et conséquemment les grammairiens, aient considéré ces deux pronominaux dans un contexte transitif.

Bruneau (1970), comme Dauzat, note l'impact des premiers grammairiens : [...] la Grammaire des grammaires connaît un succès considérable, même auprès des grands écrivains. Girault-Duvivier eut l'idée de réunir dans un corpus toutes les décisions prises par les "grammairiens éminents" des derniers siècles : Vaugelas y voisine avec Domergue, et leurs règles s'appuient sur les exemples d'écrivains “approuvés", c'est-à-dire d'auteurs classiques. (p. 26).

Pour mieux comprendre l'évolution de l'esprit grammatical, il convient de citer encore une fois Bruneau : «Il ne se publie plus, en France, d'ouvrages semblables à la Grammaire des grammaires de Girault-Duvivier ou à la Grammaire de Poitevin. Le Bon Usage de Grevisse, qui en tient lieu aujourd'hui, n'est pas un catéchisme grammatical : alors que Girault-Duvivier et Poitevin imposaient des règles, garanties par des exemples empruntés à des auteurs approuvés, Grevisse donne des exemples tirés d'auteurs de tout genre, et parfois nettement contradictoires [...]» (p. 271). Cela se confirme par les 17 cas susmentionnés dont plusieurs sont cités par Grevisse.

\section{L'accord ou l'invariabilité du participe passé dans le contexte scolaire}

Arrivé (1993) fait référence au Rapport du Conseil supérieur de la langue française sur les rectifications de l'orthographe de 1990 et cite un passage non sans importance pour les instituteurs, les enseignants et les professeurs : «L'orthographe actuelle reste d'usage, et les "recommandations" du Conseil supérieur de la langue française ne portent que sur des mots qui pourront être écrits de manière différente sans constituer des incorrections ni être jugés comme des fautes » (déclaration de l'Académie en janvier 1991) (p. 121).

En ce qui nous concerne, ce propos n'affecte que le cas $\mathrm{n}^{0} 12$ - Le participe passé avec

" avoir 》 et " fait, laissé » suivis de l'infinitif et le cas n ${ }^{0} 16-$ Le participe passé des verbes pronominaux suivis de l'infinitif. En effet, comme susmentionné dans ces mêmes cas, le Conseil supérieur a tranché la question : " garder le participe passé "laissé" suivi de l'infinitif invariable », que ce soit dans un contexte pronominal ou non pronominal. Dès lors, se pose la question de savoir dans combien de temps les enseignants appliqueront cette recommandation; autrement dit, lorsque Goosse (1991) souligne que « on observe un double usage, et pour éviter le désordre que craignent aussi les correcteurs, le Conseil a transformé la latitude en règle » (p. 70), se pose la question à savoir est-ce que les enseignants vont sanctionner une erreur si le participe passé, dans ces cas, est utilisé selon la prescription des nombreuses grammaires antérieures à cette date. Dans l'affirmative, cela est justifiable puisque c'est la nouvelle règle; toutefois, cette sanction nous semble sévère, voire préjudiciable pour les scripteurs qui connaissent plus leurs règles de grammaire et qui les appliquent selon les nuances et les subtilités antérieures. Sur ces deux cas, nous ferons ultérieurement la recommandation que nous jugeons la plus appropriée.

Quant aux autres cas, l'arrêté Haby du 28 décembre 1976 (publié dans le Journal officiel du 9 février 1977) utilise deux formules : « L'usage admet, selon l'intention, ... » et « On admettra... 
dans tous les cas ». Ainsi, selon cet arrêté, les cas où ne sera pas compté de fautes aux candidats sont :

- le cas $\mathrm{n}^{0} 2$ Le participe passé utilisé sans auxiliaire : cas de " compris, non compris, excepté, ôté, y compris » (bien que ce cas comporte plusieurs participes tels que précités, nous n'avons mentionné que ceux notés par l'arrêté). Exemples : J'aime tous les sports, excepté la boxe (EXCEPTÉE LA BOXE). J'aime tous les sports, la boxe exceptée (LA BOXE EXCEPTÉ).

- le cas $\mathrm{n}^{0} 3$ Le participe passé utilisé sans auxiliaire : cas de « ci-inclus, ci-joint 》 (seuls cas notés par l'arrêté). Exemples : Ci-inclus (CI-INCLUSE) la pièce demandée. Vous trouverez ci-inclus (CI-INCLUSE) copie de la pièce demandée. Vous trouverez cette lettre CI-INCLUSE. Vous trouverez cette lettre CI-INCLUS.

- le cas $\mathrm{n}^{0} 4$ Le participe passé utilisé sans auxiliaire : cas de "étant donné 》 (seul cas noté par l'arrêté). Exemples : ÉTANT DONNÉES les circonstances... ÉTANT DONNÉ les circonstances...

- le cas $\mathrm{n}^{0} 9$ Le participe passé avec « avoir » et « courir, coûter, dépenser, distribuer, donner, gagner, mesurer, parier, perdre, peser, prendre, rapporter, recevoir, recueillir, récolter, régner, travailler, valoir, vivre... ». Exemples : Je ne parle pas des sommes que ces travaux m'ont coûté (COÛTÉES). J'oublierai vite les peines que ce travail m'a coûtées (COÛTÉ).

- le cas $\mathrm{n}^{0} 10$ - Le participe passé avec « avoir » et suivi de l'infinitif (sauf en ce qui concerne le participe passé du verbe faire). Exemples : Les musiciens que j'ai entendus (ENTENDU) jouer.

- le cas $\mathrm{n}^{0} 13$ Le participe passé avec " avoir » et précédé du pronom " en » (dans une forme verbale précédée de en complément de cette forme verbale). Exemples : J'ai laissé sur l'arbre plus de cerises que je n'en ai CUEILLI. J'ai laissé sur l'arbre plus de cerises que je n'en ai CUEILLIES.

Si nous résumons ce qui précède dans le contexte de la pratique évaluative, le Conseil supérieur de la langue française s'est prononcé uniquement par rapport aux cas $\mathrm{n}^{0} 12$ et 16 , c'est-à-dire " garder le participe passé "laissé" invariable lorsqu'il est suivi de l'infinitif ». L'arrêté Haby, au contraire, formule des tolérances non seulement pour ces cas, mais aussi pour les cas $n^{0} 2,3,4,9$, 10 et 13. La brève incursion historique nous dévoile que, d'une part, le double usage des 17 règles n'ont pas été à l'abri de controverses entre grammairiens, surtout à partir du XVII siècle et, d'autre part, la plupart de ces règles sont fondées au départ sur la révérence des modèles littéraires. De plus, ironiquement, les grands écrivains ne sont pas exempts de reproches des grammairiens tels que Vaugelas et ce dernier est lui-même critiqué par d'autres grammairiens. Grevisse, grammairien plus contemporain, recense des exemples tirés de grands écrivains de tout genre, exemples parfois qui contredisent les règles, mais qui reflètent l'observation de l'usage et des usages. Qu'advient-il de l'évaluateur qui doit sanctionner les erreurs dans les écrits des étudiants et de ces derniers qui contestent l'évaluation?

Pour répondre à cette question, nous formulons les recommandations suivantes à la suite d'expériences vécues :

- Même si le Conseil supérieur de la langue française a transformé la latitude en règle (cas du participe passé de laisser suivi de l'infinitif), il est plus sage d'accepter cette règle (garder, dans ce cas, ce participe passé toujours invariable) sans pour autant sanctionner l'ancienne règle (accord ou non du participe passé selon que le complément d'objet direct fait l'action de l'infinitif ou non) tant qu'il existe des livres de grammaire qui présentent ces différences. Dans le cas où le correcteur 
appliquera à la lettre la recommandation du Conseil supérieur, il est de son devoir d'informer tous les étudiants de l'existence de cette règle et de la sanction qu'il compte faire si la règle n'est pas respectée.

- Les tolérances de l'arrêté Haby ne sont pas une immunité diplomatique pour tous les étudiants et encore moins pour n'importe quel scripteur, à qui il serait permis d'écrire en transgressant les règles existantes. En effet, cet arrêté concerne des

《 tolérances grammaticales ou orthographiques » dans les examens ou les concours relevant du ministère de l'Éducation (en France) et sanctionnant les étapes de la scolarité élémentaire et de la scolarité secondaire. De plus, cet arrêté mentionne clairement que «parmi les indications qui figurent ci-après, il convient de distinguer celles qui précisent l'usage et celles qui proposent des tolérances. Les premières doivent être enseignées. Les secondes ne seront prises en considération que pour la correction des examens ou concours dépendant des ministères français; elles n'ont pas à être étudiées dans les classes et encore moins à se substituer aux connaissances grammaticales et orthographiques que l'enseignement du français doit s'attacher à développer ». Donc, l'arrêté Haby n'est pas la solution. Sur ce point, Grevisse (1980) fait référence à un article de la Revue générale de mars 1977 où Hanse, avec qui il est en accord, critique cet arrêté : « ...il est dangereux et néfaste d'habituer les élèves à croire qu'on admettra dans tous les cas des licences que l'usage, non seulement littéraire mais commun, continue à proscrire...».

- Malgré les 17 cas précités, nous suggérons, au risque d'être taxé de sévère et d'impitoyable, que les règles existantes prévalent et soient respectées. Donc, à moins qu'il ne soit clairement noté que le double usage est permis, il est recommandé de ne regarder ces 17 cas que comme une fluctuation malheureuse de la norme et de s'en tenir aux règles strictes. Nous croyons que la référence aux écrits d'illustres écrivains qui enfreignent les règles ne devrait pas cautionner la politique du non-respect des règles.

- Dans une perspective juridique, l'existence du double usage dans les 17 cas en question permet à des étudiants universitaires d'avoir recours à des avocats pour tenter de modifier leurs notes à la hausse. Un tel geste s'explique par le fait que, dans certains cours, les étudiants perdent jusqu'à $20 \%$ de la note finale à cause des erreurs reliées, entre autres, à la grammaire et à l'orthographe. Dans plusieurs universités francophones du Québec, la qualité dans les écrits des étudiants est une condition sine qua non pour l'obtention du diplôme, et conséquemment de la possibilité de trouver un emploi. D'où l'importance d'avoir des règles nettes et précises confirmées par un usage non équivoque.

Après avoir mis en relief les 17 règles «problématiques » et après avoir tenté de cerner le pourquoi de cette flexibilité et ses conséquences dans la pratique évaluative, notamment dans le contexte scolaire, nous sommes en droit de nous demander si les cas précités ne sont pas le reflet d'une mentalité frileuse qui préfère se cantonner au flou énigmatique du participe passé. Cette remarque, bien que désobligeante, n'enlève rien à la richesse et à la complexité de cette notion au sujet de laquelle les débats se sont accentués à partir du XVII siècle.

Notre recherche démontre que les 17 cas susmentionnés sont le résultat de l'observation de l'usage de la langue écrite de divers écrivains par les grammairiens, écrivains au départ considérés célèbres ou approuvés, et plus tard au xxe siècle des écrivains tout court. Enfin, au risque d'être taxé de rigoureux, nous préférons des mesures moins timides pour éviter le chaos qui succède à 
une observation lâche de la langue sous prétexte que tel ou tel écrivain a respecté ou non les règles. Vaugelas avait probablement raison de critiquer certains écrivains. De nos jours, qui s'élèvera contre le double usage sans passer pour un «non-linguiste » issu d'une tradition immuable? Enfin nos modestes recommandations finales ne visent qu'à être un éclairage utile aux enseignants et professeurs dans l'évaluation des écrits de leurs étudiants bien que rien n'empêchera les étudiants riches de recourir aux avocats en brandissant l'étendard du double usage des 17 cas précédents pour faire courber l'échine aux professeurs stricts.

\section{Bibliographie sommaire}

Arrivé, Michel. Réformer l'orthographe. Paris : PUF, 1993.

Bescherelle frères et Litais de Gaux. Grammaire nationale ou Grammaire de Voltaire, de Racine, de Bossuet, de Fénelon, de J.-J. Rousseau, de Buffon, de Bernardin de Saint-Pierre, de Chateaubriand, de Casimir Delavigne. Paris : Bourgeois-Maze, 1836.

Bouhours, Dominique. Remarques nouvelles sur la langue françoise. Paris : Sébastien MabreCramoisy, 1675. Rééd. Genève : Slatkine reprints, 1972.

Bruneau, Charles. Petite histoire de la langue française. Paris : Librairie Armand Collin, 1970. Tome II, De la révolution à nos jours. $5^{\mathrm{e}}$ éd. revue et corrigée Monique Parent et Gérard Poignet.

Chaurand, Jacques. Nouvelle histoire de la langue française. Paris : Seuil, 1999.

Corneille, Thomas et al. Commentaires sur les Remarques de Vaugelas, Paris : Droz, 1687. 2 vol. Réed. Jeanne Streicher. Genève, Slatkine reprints, 1970.

Dauzat, Albert. Tableau de la langue française. Paris : Payot, 1967.

Fournier, Nathalie. Grammaire du français classique. Paris : Belin, 1999.

Girault-Duvivier. La grammaire des grammaires ou Analyse raisonnée des meilleurs traités sur la langue française. 2 vol. Éd. revue et augmentée Pierre-Auguste Lemaire, 1853.

Goosse, André. La « nouvelle orthographe ». Paris - Louvain-la-Neuve : Duculot, 1991.

Grevisse, Maurice. Le bon usage. 11 éd. revue. Paris - Gembloux : Duculot, 1980.

République française. "Arrêté Haby ». Journal officiel de la République française. 9 février 1977

République française. « Rapport du Conseil supérieur de la langue française sur les rectifications de l'orthographe ». Journal officiel de la République française. 6 décembre 1990.

Vaugelas, Claude Favre de. Remarques sur la langue françoise. Paris : Vve Jean Camusat \& Pierre Le Petit, 1647. Rééd. Jeanne Streicher. Paris : Droz, 1934. 